\title{
LASER SIMULATION Methods of Pulse Detection in Laser Simulation
}

\author{
Jana Hájková \\ Department of Computer Science and Engineering, University of West Bohemia, Univerzitni 22, Pilsen, Czech Republic \\ hajkovaj@kiv.zcu.cz
}

Keywords: Simulation, visualization, method, pulse detection, laser, application approach, automation, centre of mass.

\begin{abstract}
This paper deals with the problem of laser simulation. At the beginning it gives a broader overview of the project of laser simulation which is processed at the University of West Bohemia. The simulation is described in several fundamental steps, a technique of data obtaining, processing and usage for the simulation is highlighted to understand the whole approach well. Three methods for automatic pulse detection are described in detail. Pulse detection is the main part of the pulse extraction, which is one of the most important data processing steps. The main idea of each described method is explained and their problems and possible ways of their elimination are discussed. At the end of the paper future plans for the project with the focus on the alternatives of system automation are introduced.
\end{abstract}

\section{INTRODUCTION}

About one year ago a project started in a cooperation of several departments of University of West Bohemia - the Department of Computer Science and Engineering, the Department of Physics and the Department of Cybernetics. Also the Department of Mathematics from the same university is partly interested in the project. Except these four university departments, also a hi-tech company Lintech participates in this project and supports it.

Our aim is to develop a real laser equipment (HW device) for sophisticated laser burning into various materials. Besides the excellent $\mathrm{HW}$ components this device must have also several indispensable SW modules, which control the laser, simulate its function or enable to explore burned experiments. The basics of the whole project and its parts are detailed in (Hájková and Herout, 2008).

The task for our group is to create a SW support in the form of simulation system for offline and online controlled simulations and a sophisticated tool for exploring of burned experiments which could be used independently.

The paper is divided into six chapters. Following Section 2 describes the basic simulation process, in Section 3 methods for automatic pulse detection are worked out and in Section 4 our results are outlined. Section 5 presents out future plans and Section 6 concludes the paper.

\section{LASER SIMULATION}

The whole system of laser device should serve for miscellaneous scientific and commercial experiments. Results of these experiments are not fully deterministic, that is why they sometimes need to be reoperated several times to obtain optimal result. Repetitious burning of the same experiment is money and time consuming. That is why any software tool which would eliminate real burning of incorrect results is beneficial.

The simulation should provide experiments as quick and cheap as possible. It would also enable optimization from different points of view (e.g. speed or accuracy) and help to eliminate the unreasonable experiments. All parts of the simulation should be automatic in the maximal way so the simulation can run independently of the user. Moreover, in contrast to real burning, where each experiment requires servicing, simulation creates a possibility of batch-oriented experiments executing. After the simulation finishes, the best results can be selected and all gained results are described.

As a part of the simulation there should be also implemented a tool for data 2D and 3D visualization. This tool would enable to explore real or simulated results and to interpret accuracy and optimality of the simulated sample.

Each simulation has to be based on the simulation model. We decided to use an application 
approach; it means that our simulation model comes out from real data measured from real burned experiment.

\subsection{Data Description}

At first, the way of data obtaining should be described. To get any data for the simulation input, samples have to be burned by existing laser equipment into the real material. After burning the real samples, they have to be measured. For this purpose the confocal microscope Olympus LEXT OLS3100 is used.

The sample surface is represented by the matrix of floats expressing heights in corner points of the uniform rectangular grid. This grid represents a height map which describes the surface of the sample.

The majority of samples which we use have the same form. The burned pulse fills preponderance of the sample surface; the real dimension of the sample is $256 \times 192 \mu \mathrm{m}$. The grid of height map is really fine; most common grid step in used data is $0.25 \mu \mathrm{m}$. It means that the surface of such sample is described by $1024 \times 768$ values. We dispose with samples burned into two materials: steel and cermet (a composite material composed of ceramic and metallic materials).

\subsubsection{Pulse Representation}

Let's explore the pulse in a detail, at first with a little bit simplification. In an ideal situation the surface of the material would be perfectly smooth without any roughness. When the laser burns one pulse into such material, it will modify the surface, a hollow in the point of burning will be created and around the hollow the melted material will make a bulge (as seen in Figure 1a). By these conditions if we place two laser pulses at almost one place, we can use a principle of summation. Result is shown in the Figure $1 \mathrm{~b}$ where two pulses next to each other are placed.

But in the real environment we can hardly expect ideal conditions. Because we use real data which are measured with high zoom, roughness of the material plays quite important role and the surface roughness causes inconsiderable problems during the sample processing. Cross-sections of real pulse (1 pulse burned into the cermet) can be seen in Figure 2 .

At the beginning of our work it was important to choose any suitable form of pulse representation. At first we planned to represent the pulse by several parameters or by its cross-section. The whole pulse should be gained as a surface of revolution. It means that the surface of the sample would be obtained by rotating the curve of cross-section in space about an axis passing throw the middle. This method could be used in the case of ideal situation, but in the case of real samples this representation of the pulse would mean unacceptable distortion of data. a)

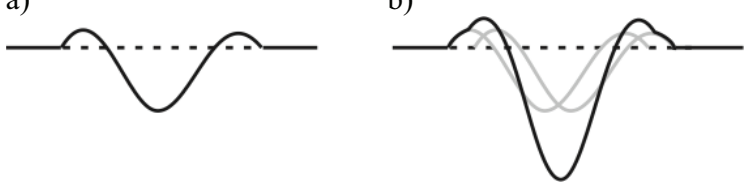

Figure 1: Simplified result of burning into ideally smooth material a) one pulse b) two pulses next to each other.

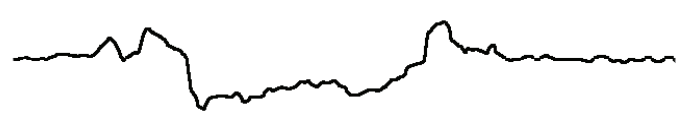

Figure 2: Cross-section of real pulse.

That is why we had to search for another way of pulse representation. Finally we decided for the form of height map representation of the whole pulse surface. There was a question how to describe the values of the pulse surface to be used for the burning simulation in the simplest way. From several experiments we decided to represent the level of the material by the value of zero. All points of the sample height map are during pulse extraction recounted and saved as differences of the surface of the material and their original height. It means that the final saved pulse consists of positive and negative real values. The positive values represent material upon the basic material level; on the contrary the negative ones represent material which has vaporized.

The format of height map is exact enough, but its disadvantage embodies in amount of data files which have to be saved for various pulses for different materials. The size of the file is not very high but we still speculate about the redundancy of this format. That is why we would like to try another method of pulse representation.

Pulses are extracted from input samples. Because of high amount of input samples it is necessary to make the process of pulse extraction automatic. The main part of the process is created by the automatic pulse detection. Because it is the main topic of this paper, it is particularized separately in Section 3. 


\subsection{Simulation Approach}

As it was described in (Hájková and Herout, 2008), we decided to use for the simulation the application approach.

The pulses are extracted from input data for given combination of used material and laser setting. The basic technique of simulation of samples burning is to place selected pulses gained from input data on the surface of the unburned material. The format of the pulse is designed for the simplest usage as possible and it offers the ability of direct pulse application on the surface of the material (as it was described in the previous sections).

The simulation itself requires solving of many problems (e.g. heating of the material during repetitive burning of laser ray into one point of the material, influence of the material surface roughness on the laser ray reflection or inaccuracies caused by starting and finishing laser motion).

For the correct function of the simulation the system has to be verified. During the verification the burned samples are compared with the real ones, which are gained by the same method as it was described in Section 2.1. To test and evaluate the system in a more global way a broad range of samples has to be simulated and compared. An automatic verification is used for speeding it up.

\section{METHODS FOR AUTOMATIC PULSE DETECTION}

Pulse detection is used as a part of data preprocessing. The task of detection is to define the area of the material surface which was affected by the laser burning as exactly as possible. Pulse detection can be done of course manually but for the speedup and simplification of the whole preparation process, the system has to prepare maximum of parts self-containedly. But the precision and accuracy has to be preserved as well with the automation process. That is why we have to develop appropriate algorithm for pulse detection.

The main problem is the roughness of the basic material surface. As it can be seen in Figure 3 for some materials, such as for a steel in Figure $3 \mathrm{a}$, the surface is quite smooth. Another situation comes in the case of cermet (Figure $3 b$ ), where the protrusions on the material surface are more noticeable. Moreover, for all types of material also local roughnesses which are not specific for the material can appear. Such defects need not to be visible on the material surface by naked eye, but thanks to the height resolution of real sample scanning, they are included in the description of the sample and are the source of problems during the pulse auto-detection. Local roughness can be seen in Figure $3 \mathrm{a}$ on the upper side of the sample.
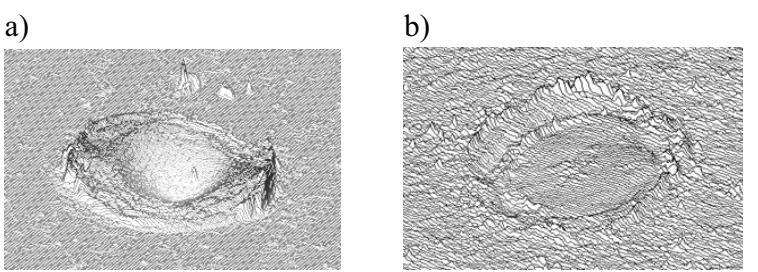

Figure 3: a) Surface of steel with relatively smooth surface; local defect can be seen on the upper side of the sample. b) Surface of cermet sample with globally higher roughness of the surface.

The user is able to distinguish roughness or defects of the material during the manual pulse detection, but for the automatic method it is very difficult to differentiate these inaccuracies from the border of the pulse. Chosen methods have to be precise enough, but very precise methods are already slow. Quickly working methods are unfortunately inaccurate. That is why we have to find a compromise and to create a new method designed right for this task.

\subsection{Global Extremes Method}

The first version of detection algorithm goes from unmodified sample surface. The algorithm starts from points with the minimal and maximal height. These points are supposed to be in the area of the burned pulse that should be detected. From their position columns of height map to the left and to the right side are inspected and the height difference of points in each single column (it means the difference between the maximal and minimal value in the column) is counted. If the value does not exceed given height limit, an inspection in the direction is finished. After cutting of columns on the left and right side of the pulse, the same process of border searching is started for rows. Horizontal borders are appointed.

There is a question how to gain the value of difference height limit. This is one of problems of this algorithm. If we do not want to set the constant manually we have to explore the sample automatically, for example during its loading into the system. We can suppose that borders of the sample are not modified by the burning and that is why they represent the original material surface and 
so the difference constant can be for most samples precounted, for example as the minimal height difference counted from several border columns. But this approach may not work well if the used border of the sample is damaged by any local defect of the material. In such case the difference constants is counted too high.

The worse problem that causes wrong results of this method is brought by local defects of large height or depth. The defect can move the location of global minimum or maximum from the area of the pulse to the area of local defect. This problem can be solved by changing of the algorithm for the starting point searching.

\subsection{Centre of Mass Method}

Another way how to get the starting point for automatic pulse detection is to find the position of centre of mass in the sample. The typical procedure of the center of mass computation has to be adapted for the sample representation. Basic expression for calculation of the center of mass $x_{c}$ of a system of particles is defined as the average of the particle positions $x_{i}$, weighted by their masses $m_{i}(1)$.

$$
x_{c}=\frac{\sum m_{i} \cdot x_{i}}{\sum m_{i}}
$$

Let us label the point of the sample as $f_{i}=f[x, y]$. Because the whole sample is represented by positive values (the values representing the material level in the sample are given by the height of the material into which the sample was burned), we could define $m_{i}=f_{i}$. The simplified cross-section curve of the sample can be seen in Figure 4a. The result is shown in Figure 5a. If the pulse does not take the majority space of the sample and the pulse is not placed in the centre of the sample, the rest of the surface overbalances the pulse area and the centre of mass is moved from the centre of the pulse partly to the side of plain surface.

That is why it is necessary to shift the whole sample so that the material level is represented as a zero value. By this shifting some parts of the sample are represented by the negative value. Such sample cannot be used for center of mass computation and so the negative values have to be converted to positive ones. That can be done by using power function with even and positive exponent. To stress values of the pulse from small values in the neighbourhood of the zero level, we decided to use exponent 4 (Figure 4b). Finally the weights for center of mass computation are defined as in the expression
(2), where basicLevel represents the height of the basic material. The result of such calculation can be seen in Figure $5 b$.

$$
m_{i}=\left(f_{i}-\text { basicLevel }\right)^{4}
$$

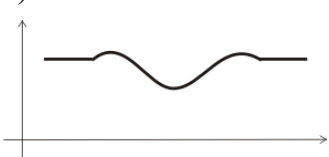

b)

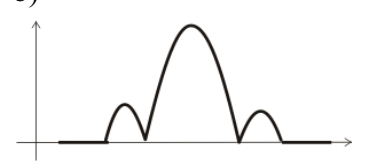

Figure 4: Simplified cross-section of the sample in all both phases of computation; a) the original sample, b) the sample after application of power function. a)

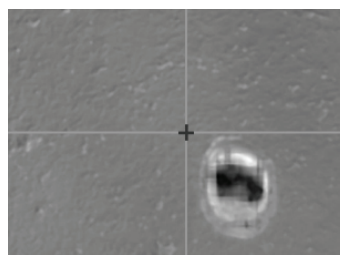

b)

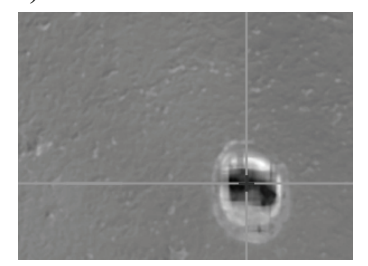

Figure 5: a) The result of the centre of mass computation with the weight $m_{i}=f_{i}$. b) The result of the centre of mass computation with the weight $m_{i}=\left(f_{i} \text {-basicLevel }\right)^{4}$.

By this method we get the starting position which is placed in the area of burned pulse and is affected by local material defect in a minimal way. The other steps of algorithm described in Section 3.1 can be used in this time or we can choose another technique which is described in Section 3.3.

\subsection{Spiral Method}

During testing the location of starting point was always found correctly in the area of burned pulse for all samples we dispose with. So we can try to use another approach for finding borders of the pulse (previously described algorithm of pulse borders detection is sensitive to local defects on the material surface). We can start in the starting point and then inspect the surroundings up to finding the basic level of the material.

In the ideal case, the pulse has circular or ellipsoidal shape and the centre of mass is placed exactly in the centre of the pulse. For such pulse we can find the bounding rectangle simply. If we put through the centre of mass two lines parallel with axis $x$ and $y$ (as can be seen in Figure 6a), we can make cross-sections of the sample along these lines (the vertical and the horizontal ones).

The cross-section curve is optimally in the area of the pulse more diverted than in the part of the 
unburned material. That is why we can determine two points of the curve, where the pulse finishes and to define borders there (Figure $6 \mathrm{~b}$ ).

a)

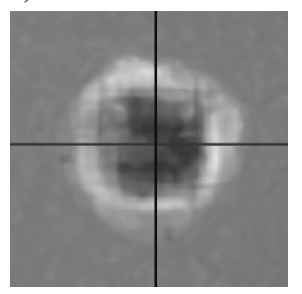

b)

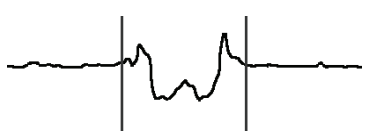

Figure 6: a) Sample with nearly circular shape; two lines parallel with axis $x$ and $y$ are going through the centre of mass. b) Cross-section curve with defined borders.

Searching of border values starts at the beginning and at the end of the curve and continues in the direction to the centre of mass. First, we are in the area of unburned material where values of the curve do not differ from the average material height a lot. When the values start to differ more we have found the border of the pulse. To prevent mistakes caused by roughness of the material, the same height limit, as in the algorithm described in Section 3.1 (gained during the sample loading), is used.

In the real cases the method described above is not sufficient, but we can use its result as a starting state for the next processing. If the shape of the pulse is irregular, the centre of mass is shifted from the middle of the pulse (as in Figure 7a). Moreover, the irregularity of the pulse from the top view deflects the borders (as in Figure 7b). That is why the previous procedure gives only a rectangle that borders a part of the pulse.

a)

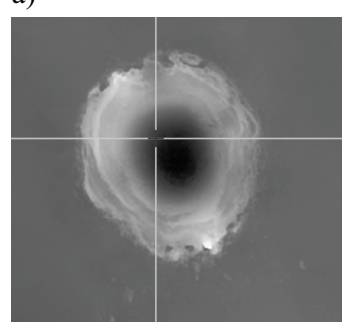

b)

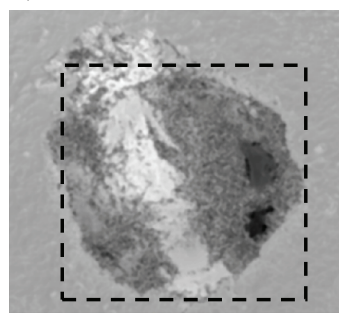

Figure 7: a) Shifted position of the centre of mass location. b) Asymmetry of the pulse shape in the top part of the pulse from the top view that will cause top border shifting. The border determined by the algorithm is dashed.

The final borders are searched in a spiral way. All borders are periodically tested if it is possible to move them for one row or one column further from the centre of mass. In each step for each single border (left, bottom, right and top) the height difference between the minimal and the maximal value in the shifted position is computed and compared with the difference limit for the processed sample. The sequence of borders is preserved through the whole computation (it means borders are rotating during the algorithm). If any border can not be moved it is not used any more in following steps.

\section{RESULTS}

To describe all results of existing SW part of the project, a highly specialized tool which was designed and subsequently also implemented would have to be mentioned, because there was no available tool for data exploring and modification. Because this paper is focused on the topic of automatic pulse detection methods so this section will concentrate only on results of these methods.

Several methods for pulse detection were described. All methods were tested on the same samples which were chosen because of any typical feature. The task of the algorithm has been to detect the pulse in the most perfect way. In this section problematical samples are described and discussed. Some of tested samples are burned into cermet, where the high roughness of the material can influence the detection and some are burned into steel which has much smoother surface. Samples with various counts of laser pulses burned into one point of the material were chosen. Surfaces of several samples are influenced by the local defects of the material and shapes of pulses are in some cases more and in some less asymmetric.

A feature which is problematical for all methods is the determination of height difference limit. The way of precounting the value as the minimal height difference counted from several border columns works quite well for the samples without local defect on the material surface. But the areal defect around the pulse can produce incorrect pulse detection as it is shown in Figure 9a.

Another problem is the roughness of the material surface for example in the case of cermet. Each of methods copes with it in a little bit different way. Results of the Centre of mass method are shown in Figure $8 \mathrm{a}$ and it can be compared with the result of the Spiral method in Figure 8b. The value of height difference limit was computed too high because of the roughness of the material. In the first case where left and right border were found first, part of the pulse was not bordered. In the second case, thanks to the spiral approach, this did not happen at the cost of expansion of bordering rectangle. 
a)

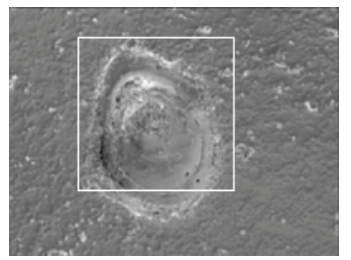

b)

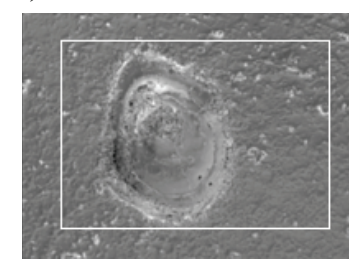

Figure 8: Comparison of pulse detection in the sample with high roughness of the material - a) Centre of mass method; b) Spiral method.

The main disadvantage of the method starting from global extremes is in the setting of the starting position. If there is any local defect of extreme height, the starting point for pulse border searching is shifted into the position of local defect and the result is distorted. The sample corresponding to such situation can be seen in Figure 9b.

a)
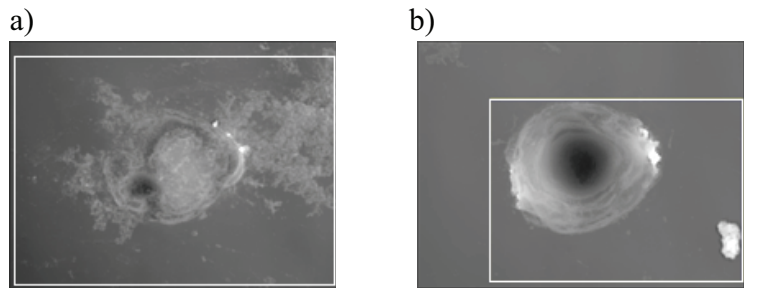

Figure 9: Problematic samples with local defects a) of areal character around the pulse which causes incorrect pulse detection; b) of extreme height in the surrounding of the sample which causes wrong localization of starting position.

\section{FUTURE PLANS}

Our future plans are divided into several groups in dependence to which activity it is related to. Of course, one of our most important aims is to improve the simulation to get as realistic results as possible. But with regards to the main topic of this paper let us focus on our plans concerning improvements of program self-activity.

The whole system should work as automatic as possible. That is why all partial tasks of data processing should be self-acting if possible. Of course, despite the automation, the reliability and speed of the system must be preserved.

All methods for pulse detection described above use similar principles and that is why they face the similar problems. So we decided to try to test another approaches. One of the tested alternatives are methods used for image processing and recognition such as thresholding, erosion, dilatation, edge detection, pattern recognition, various types of image filtration etc. (Hlaváč and Šoula), (Hlaváč and Sedláček). These methods are in the process of testing for the present and they are not used for the detection itself yet. Methods are tested in combination with different variants of resampling of the image. The results look well so far, the main problem is the automaticity of the detection process, because for each sample the setting is different.

Another way to automatic pulse detection might be methods using linear and non-linear diffusion filtering (Nishiguchi, Imiya and Sakai) in the combination with isolines searching. Also this approach is in the phase of testing.

\section{CONCLUSIONS}

The project is in the phase of partial completion. We have prepared a tool for data preprocessing and groundwork for simulation tool. It enables us to provide various experiments. For this purpose we dispose with data sets from experiments burned and measured for two materials - cermet and steel.

In the close future we plan to maximize the self-activity of the pulse detection tool and we want to improve methods for system verification and results comparison.

\section{ACKNOWLEDGEMENTS}

The work was supported by the Ministry of Education, Youth and Sport of the Czech Republic "University spec. research -1311 ".

\section{REFERENCES}

Blelloch, E. G., 2001. Introduction to Data Compression [online], [cit. 2008-03-28]. $<$ http://www.cs.cmu.edu/afs/cs/project/pscicoguyb/realworld/www/compression.pdf $>$

Hájková, J., Herout P., 2008. Laser Simulation. Proceedings of the 7th International Conference APLIMAT 2008, STU Bratislava.

Hlaváč, V., Šoula, M., 1994. Image Processing and Machine Vision, Chapman \& Hall Computing, London.

Hlaváč, V., Sedláček, M., 2001. Zpracování signálů a obrazů, Vydavatelství Č́VUT. Praha.

Nishiguchi, H., Imiya, A., Sakai, T., 2006. Laplacian Based Non-Linear Diffusion Filtering. Proceedings of 18th International Conference on Pattern Recognition ICPR 2006. 